\title{
Effect of Radical Species and Operating Parameters on the Degradation of Sulfapyridine Using UV/Chlorine System
}

Huaying Liu, Biaojun Zhang, Yingjie Li*, Qi Fang, Zhichao Hou, Senlin Tian*, Junjie Gu Faculty of Environmental Science and Engineering, Kunming University of Science and Technology, Kunming, Yunnan, China 650500

Text S1 Detailed analytical processes for water matrices.

Text S2 Determination of $k_{\mathrm{SPY}, \mathrm{OH}}$

Text S3 Determination of $k_{\mathrm{SPY}, \mathrm{Cl}}$ and $k_{\mathrm{SPY}, \mathrm{Cl} 2}$.

Text S4 Determination of $k_{\mathrm{SPY}, \mathrm{CO} 3-}$

Table S1 Sulfapyridine and its associated substructure analogues

Table S2 pH, TOC, and concentrations of ions for the freshwater and seawater

Table S3 Factor definitions, coding levels, and design points for the four-factor central composite design

Experimental parameters and the degradation rate constants for sulfapyridine in $\mathrm{UV} /$ chlorine system

Table S5 HPLC parameters for sulfapyridine and its corresponding substructures

Table S6 Accurate mass of the degradation products for sulfapyridine in UV/chlorine oxidation system

Figure S3

Evolution profiles of the degradation products of sulfapyridine under $\mathrm{UV} /$ chlorine system

Figure S4

3D response surface graphs of pseudo-first order degradation rate constant $\mathrm{k}$

Figure S1 Degradation of sulfapyridine and nitrobenzene by $\mathrm{UV} / \mathrm{H}_{2} \mathrm{O}_{2}$ oxidation process

Figure S2 Degradation of sulfapyridine, benzoic acid, and nitrobenzene by UV/chlorine

Figure S5 Degradation of sulfapyridine, benzoic acid, and nitrobenzene by UV/chlorine

*Corresponding author: e-mail: yjli@kmust.edu.cn; tiansenlin@outlook.com 
oxidation process containing $0.3 \mathrm{M} \mathrm{Cl}^{-}$

Figure S6

Kinetic plot of sulfapyridine (SPY) and salbutamol for the determination of the second-order rate constants of $\mathrm{CO}_{3} \cdot \cdot$ reacting with sulfapyridine

Figure S7 Degradation kinetics of sulfapyridine by UV/chlorine process in synthetic water and effluent water samples

\section{Text S1 Detailed Analytical Processes for Water Matrices}

The $\mathrm{pH}$ and conductivity of water samples were measured by a pH-meter (Shanghai Rex, PHS-3E). The conentrations of total organic carbon (TOC) for humic acid solution and real water samples were determined by a TOC analyzer (Hangzhou Qikun, CD-800S) after the water samples were acidified with $1 \mathrm{M} \mathrm{HCl}$ and purged with nitrogen. Anion and cation concentrations were measured by a ThermoFisher ion chromatography (DIONEX ICS-5000). The concentrations of $\mathrm{HCO}_{3}{ }^{-}$were determined by the Chinese standard GB/T 8538-2008 method.

\section{Text S2 Determination of $\boldsymbol{k}_{\mathrm{SPY}, \mathrm{OH}}$}

The second-order rate constant of sulfapyridine (SPY) with $\cdot \mathrm{OH}\left(k_{\mathrm{SPY}, \mathrm{OH}}\right)$ was determined by competition kinetic method. To determine the $k_{\mathrm{SPY} \text {, OH }}$ value between $\cdot \mathrm{OH}$ and sulfapyridine, a solution containing $20 \mu \mathrm{M}$ target compound, $20 \mu \mathrm{M}$ nitrobenzene $\left(\mathrm{NB}\right.$, a probe of $\cdot \mathrm{OH}, k_{\mathrm{NB}, \cdot \mathrm{OH}}$ $\left.=3.9 \times 10^{9} \mathrm{M}^{-1} \mathrm{~s}^{-1}\right),{ }^{\mathrm{S} 1}$ and $100 \mathrm{mM} \mathrm{H} \mathrm{H}_{2} \mathrm{O}_{2}$ employed to produce $\cdot \mathrm{OH}$ was photolyzed simultaneously. The value of $k_{\mathrm{SPY}, \mathrm{OH}}$ was obtained according to the following equation:

$$
k_{\mathrm{SPY}, \mathrm{OH}}=\frac{\ln \left([\mathrm{SPY}]_{\mathrm{t}} /[\mathrm{SPY}]_{0}\right)}{\ln \left([\mathrm{NB}]_{\mathrm{t}} /[\mathrm{NB}]_{0}\right)} k_{\mathrm{NB}, \mathrm{OH}}
$$

\section{Text S3 Determination of $k_{\mathrm{SPY}, \mathrm{Cl}}$ and $k_{\mathrm{SPY}, \mathrm{Cl2}{ }^{-}}$}

The competition kinetic method was also employed to calculate the second-order rate constant of sulfapyridine with $\mathrm{Cl} \cdot$ and $\mathrm{Cl}_{2} \cdot{ }^{-}$. For the determination of $k_{\mathrm{SPY}, \mathrm{Cl}}$, a UV/chlorine 
solution consists of sulfapyridine (SPY), nitrobenzene (NB), and benzoic acid (BA, reaction with both $\cdot \mathrm{OH}$ and $\mathrm{Cl} \cdot$ ). The steady state concentration of $\cdot \mathrm{OH}$ in this system was calculated by the following equation:

$$
[\cdot \mathrm{OH}]_{\mathrm{ss}}=\frac{k_{\mathrm{obs}, \mathrm{NB}}}{k_{\mathrm{NB}, \mathrm{OH}}}
$$

where $k_{\mathrm{obs}, \mathrm{NB}}$ is the observed degradation rate constant induced by $\cdot \mathrm{OH}$. According to this result, the steady state concentration of $\mathrm{Cl} \cdot$ was calculated based on the degradation of benzoic acid subtracting the contribution of $\cdot \mathrm{OH}$, which can be expressed by the equation:

$$
[\mathrm{Cl} \cdot]_{\mathrm{ss}}=\frac{k_{\mathrm{obs}, \mathrm{BA}}-k_{\mathrm{obs}, \mathrm{NB}}}{k_{\mathrm{BA}, \mathrm{Cl}}}
$$

where $k_{\mathrm{obs}, \mathrm{BA}}$ is the observed degradation rate constant of benzoic acid, $k_{\mathrm{BA}, \mathrm{Cl}}$. is the secondorder rate constant of benzoic acid with $\mathrm{Cl} \cdot$. From these results, the degradation of sulfapyridine by UV/chlorine oxidation process can be expressed by equation (S4). Therefore, the $k_{\mathrm{SPY}, \mathrm{Cl}}$. value can be obtained by this equation.

$$
k_{\mathrm{obs}, \mathrm{SPY}}=k_{\mathrm{SPY}, \mathrm{OH}}[\cdot \mathrm{OH}]_{\mathrm{SS}}+k_{\mathrm{SPY}, \mathrm{Cl}}[\mathrm{Cl} \cdot]_{\mathrm{SS}}
$$

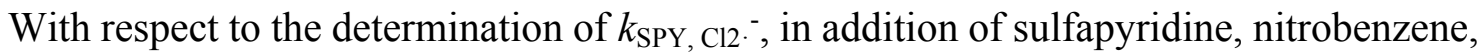
and benzoic acid in a UV/chlorine solution, $0.3 \mathrm{M} \mathrm{Cl}^{-}$was also contained in this oxidation system. In the $\mathrm{UV} / \mathrm{chlorine}$ system containing $\mathrm{Cl}^{-}, \mathrm{Cl}_{2} \cdot{ }^{-}$can be formed rapidly by the reaction of $\mathrm{Cl} \cdot$ and $\mathrm{Cl}^{-}\left(8.5 \times 10^{9} \mathrm{M}^{-1} \mathrm{~s}^{-1}\right) .{ }^{\mathrm{S} 1}$ Previous studies reported nitrobenzene can react with $\cdot \mathrm{OH}$ solely, and benzoic acid can react with both $\cdot \mathrm{OH}$ and $\cdot \mathrm{Cl}^{\mathrm{S} 1, \mathrm{~S} 2}$ Therefore, nitrobenzene was used to probe $\cdot \mathrm{OH}$, and benzoic was used to quench probe $\cdot \mathrm{OH}$ and $\mathrm{Cl}_{2}{ }^{--}$. The specific calculation processes were similar with the determination of $k_{\mathrm{SPY}, \mathrm{Cl}} \cdot$

\section{Text S4 Determination of $\boldsymbol{k}_{\mathrm{SPY}, \mathrm{CO}-}$}

The second-order rate constant for the reaction of $\mathrm{CO}_{3} \cdot-$ with sulfapyridine (SPY) was determined from the competition kinetics using salbutamol (SAL) as a reference compound, 
which reacts with $\mathrm{CO}_{3}{ }^{--}$at second-order rate constant of $2.1 \times 10^{7} \mathrm{M}^{-1} \mathrm{~s}^{-1} .{ }^{\mathrm{S} 3}$ A scenario for $\mathrm{CO}_{3}{ }^{--}$was created in the $\mathrm{UV} / \mathrm{H}_{2} \mathrm{O}_{2}$ process at a $\mathrm{H}_{2} \mathrm{O}_{2}$ dosage of $100 \mathrm{mM}$, and the solution was spiked with $100 \mathrm{mM} \mathrm{HCO}_{3}{ }^{-}$to scavenge $\mathrm{HO} \cdot$ for the formation of $\mathrm{CO}_{3}{ }^{--}$. The second-order rate constants of SPY with $\mathrm{CO}_{3} \cdot-\left(k_{\mathrm{SPY}, \mathrm{CO} 3 \cdot-)}\right)$ was calculated from the following equation:

$$
\ln \left(\frac{[\mathrm{SPY}]_{0}}{[\mathrm{SPY}]}\right)=\frac{k_{\mathrm{SPY}, \mathrm{CO}_{3}^{--}}}{k_{\mathrm{SAL}, \mathrm{CO}_{3}^{--}}} \times \ln \left(\frac{[\mathrm{SAL}]_{0}}{[\mathrm{SAL}]}\right)
$$

Table S1. Sulfapyridine and its associated substructure analogues

Compounds

Table S2. pH, TOC, and concentrations of ions for the freshwater and seawater

\begin{tabular}{cccccccc}
\hline Parameters & $\mathrm{Cl}^{-}(\mathrm{mg} / \mathrm{L})$ & $\begin{array}{c}\mathrm{Br}^{-} \\
(\mathrm{mg} / \mathrm{L})\end{array}$ & $\begin{array}{c}\mathrm{HCO}_{3}^{-} \\
(\mathrm{mg} / \mathrm{L})\end{array}$ & $\begin{array}{c}\mathrm{SO}_{4}^{2-} \\
(\mathrm{mg} / \mathrm{L})\end{array}$ & $\begin{array}{c}\mathrm{NO}_{3}^{-} \\
(\mathrm{mg} / \mathrm{L})\end{array}$ & $\begin{array}{c}\mathrm{TOC} \\
(\mathrm{mg} \mathrm{C} / \mathrm{L})\end{array}$ & $\mathrm{pH}$ \\
\hline $\begin{array}{c}\text { Freshwater } \\
\text { Coastal } \\
\text { seawater }\end{array}$ & 28.02 & $\mathrm{NA}$ & 65.36 & 51.61 & 2.64 & 30.55 & 9.76 \\
$\begin{array}{c}10.22 \diamond 10^{3} \\
\text { Wastewater }\end{array}$ & 77.90 & 97.40 & $2.52 \diamond 10^{3}$ & 59.80 & 17.93 & 8.29 \\
\hline
\end{tabular}

Table S3 Factor definitions, coding levels, and design points for the four-factor central 
composite design

\begin{tabular}{cccccc}
\hline Factors & \multicolumn{5}{c}{ Actual concentration levels } \\
\hline Coded factor levels & -2 & -1 & 0 & 1 & 2 \\
$\mathrm{pH}$ & 4 & 5.5 & 7 & 8.5 & 10 \\
$\mathrm{HA}(\mathrm{mg} / \mathrm{L})$ & 0 & 5 & 10 & 15 & 20 \\
$\mathrm{HCO}_{3}^{-}(\mathrm{mmol} / \mathrm{L})$ & 0 & 2.5 & 5 & 7.5 & 10 \\
$\mathrm{Cl}^{-}(\mathrm{mol} / \mathrm{L})$ & 0 & 0.15 & 0.3 & 0.45 & 0.6 \\
\hline
\end{tabular}

Table S4 Experimental parameters and the degradation rate constants for sulfapyridine in UV/chlorine system

\begin{tabular}{|c|c|c|c|c|c|}
\hline Runs & $\mathrm{pH}$ & $\begin{array}{c}\text { HA } \\
(\mathrm{mg} / \mathrm{L})\end{array}$ & $\begin{array}{l}\mathrm{HCO}_{3}^{-} \\
(\mathrm{mM})\end{array}$ & $\begin{array}{l}\mathrm{Cl}^{-} \\
(\mathrm{M})\end{array}$ & $\begin{array}{c}k \\
\left(10^{-2} \mathrm{~min}^{-1}\right)\end{array}$ \\
\hline 1 & 7 & 10 & 5 & 0 & $0.33 \pm 0.03$ \\
\hline 2 & 5.5 & 15 & 7.5 & 0.45 & $0.27 \pm 0.01$ \\
\hline 3 & 5.5 & 15 & 7.5 & 0.15 & $0.34 \pm 0.04$ \\
\hline 4 & 8.5 & 15 & 7.5 & 0.15 & $0.22 \pm 0.02$ \\
\hline 5 & 7 & 10 & 5 & 0.3 & $0.30 \pm 0.02$ \\
\hline 6 & 8.5 & 15 & 2.5 & 0.45 & $0.16 \pm 0.02$ \\
\hline 7 & 7 & 10 & 5 & 0.3 & $0.30 \pm 0.02$ \\
\hline 8 & 7 & 10 & 0 & 0.3 & $0.36 \pm 0.03$ \\
\hline 9 & 7 & 10 & 5 & 0.3 & $0.30 \pm 0.02$ \\
\hline 10 & 8.5 & 5 & 7.5 & 0.15 & $0.43 \pm 0.05$ \\
\hline 11 & 7 & 0 & 5 & 0.3 & $0.84 \pm 0.04$ \\
\hline 12 & 8.5 & 5 & 7.5 & 0.45 & $0.41 \pm 0.01$ \\
\hline 13 & 7 & 10 & 5 & 0.3 & $0.30 \pm 0.02$ \\
\hline 14 & 5.5 & 5 & 7.5 & 0.15 & $0.78 \pm 0.03$ \\
\hline 15 & 5.5 & 5 & 2.5 & 0.45 & $0.83 \pm 0.06$ \\
\hline 16 & 7 & 10 & 5 & 0.6 & $0.19 \pm 0.02$ \\
\hline 17 & 5.5 & 15 & 2.5 & 0.15 & $0.40 \pm 0.01$ \\
\hline 18 & 8.5 & 15 & 2.5 & 0.15 & $0.27 \pm 0.01$ \\
\hline 19 & 5.5 & 5 & 7.5 & 0.45 & $0.65 \pm 0.02$ \\
\hline
\end{tabular}




\begin{tabular}{lccccc}
\hline 20 & 7 & 10 & 5 & 0.3 & $0.30 \pm 0.02$ \\
21 & 7 & 20 & 5 & 0.3 & $0.12 \pm 0.03$ \\
22 & 4 & 10 & 5 & 0.3 & $0.90 \pm 0.04$ \\
23 & 8.5 & 15 & 7.5 & 0.45 & $0.16 \pm 0.03$ \\
24 & 8.5 & 5 & 2.5 & 0.45 & $0.40 \pm 0.01$ \\
25 & 7 & 10 & 5 & 0.3 & $0.30 \pm 0.02$ \\
26 & 5.5 & 15 & 2.5 & 0.45 & $0.32 \pm 0.04$ \\
27 & 5.5 & 5 & 2.5 & 0.15 & $0.93 \pm 0.03$ \\
28 & 10 & 10 & 5 & 0.3 & $0.09 \pm 0.02$ \\
29 & 7 & 10 & 10 & 0.3 & $0.22 \pm 0.01$ \\
30 & 8.5 & 5 & 2.5 & 0.15 & $0.51 \pm 0.03$ \\
\hline
\end{tabular}

Table S5. HPLC parameters for sulfapyridine and its corresponding substructures

\begin{tabular}{|c|c|c|c|}
\hline Compounds & $\begin{array}{l}\text { Mobile phase composition: aqueous } \\
\text { ammonium acetate }(\mathrm{pH}=5) \text { : } \\
\text { acetonitrile }(\mathrm{v}: \mathrm{v})\end{array}$ & $\begin{array}{c}\text { Detection } \\
\text { wavelength }(\mathrm{nm})\end{array}$ & $\begin{array}{l}\text { Flow rate } \\
(\mathrm{mL} / \mathrm{min})\end{array}$ \\
\hline Sulfapyridine & $80: 20$ & 260 & 1.0 \\
\hline Aniline & $80: 20$ & 234 & 1.0 \\
\hline Sulfanilic acid & 100:0 & 247 & 1.0 \\
\hline Sulfanilamide & 100:0 & 257 & 1.0 \\
\hline 2-aminopyridine & $99: 1$ & 229 & 1.0 \\
\hline
\end{tabular}

Table S6 Accurate mass of the degradation products for sulfapyridine in UV/chlorine oxidation system determined by HPLC Q-ToF mass spectrometer

\begin{tabular}{ccccc}
\hline Compounds & $\begin{array}{c}\text { Proposed formula } \\
{\left[\mathrm{M}+\mathrm{H}^{+}\right]}\end{array}$ & $\begin{array}{c}\text { Observed mass } \\
(\mathrm{m} / \mathrm{z})\end{array}$ & $\begin{array}{c}\text { Calculated mass } \\
(\mathrm{m} / \mathrm{z})\end{array}$ & Error \\
\hline $\mathrm{P} 250$ & $\mathrm{C}_{11} \mathrm{H}_{12} \mathrm{~N}_{3} \mathrm{O}_{2} \mathrm{~S}$ & 250.0642 & 250.0650 & 3.2 \\
\hline $\mathrm{P} 186$ & $\mathrm{C}_{11} \mathrm{H}_{12} \mathrm{~N}_{3}$ & 186.1015 & 186.1031 & 8.6 \\
\hline P94 & $\mathrm{C}_{6} \mathrm{H}_{8} \mathrm{~N}$ & 94.0662 & 94.0657 & -5.3 \\
P95 & $\mathrm{C}_{5} \mathrm{H}_{7} \mathrm{~N}_{2}$ & 95.0605 & 95.0609 & -4.2 \\
\hline P202 & $\mathrm{C}_{11} \mathrm{H}_{12} \mathrm{~N}_{3} \mathrm{O}$ & 202.0945 & 202.0980 & 17.3 \\
P94 & $\mathrm{C}_{6} \mathrm{H}_{8} \mathrm{~N}$ & 94.0662 & 94.0657 & -5.3 \\
\hline
\end{tabular}




\begin{tabular}{|c|c|c|c|c|}
\hline P95 & $\mathrm{C}_{5} \mathrm{H}_{7} \mathrm{~N}_{2}$ & 95.0605 & 95.0609 & -4.2 \\
\hline P110 & $\mathrm{C}_{6} \mathrm{H}_{8} \mathrm{NO}$ & 110.0598 & 110.0606 & 7.3 \\
\hline P111 & $\mathrm{C}_{5} \mathrm{H}_{7} \mathrm{~N}_{2} \mathrm{O}$ & 111.0556 & 111.0558 & 1.8 \\
\hline $\mathrm{P} 264$ & $\mathrm{C}_{11} \mathrm{H}_{10} \mathrm{~N}_{3} \mathrm{O}_{3} \mathrm{~S}$ & 264.0431 & 264.0443 & 4.6 \\
\hline P108 & $\mathrm{C}_{6} \mathrm{H}_{6} \mathrm{NO}$ & 108.0445 & 108.0449 & 3.7 \\
\hline P95 & $\mathrm{C}_{5} \mathrm{H}_{7} \mathrm{~N}_{2}$ & 95.0605 & 95.0609 & -4.2 \\
\hline P266 & $\mathrm{C}_{11} \mathrm{H}_{11} \mathrm{~N}_{3} \mathrm{O}_{3} \mathrm{~S}$ & 266.0581 & 266.0599 & 6.8 \\
\hline P94 & $\mathrm{C}_{6} \mathrm{H}_{8} \mathrm{~N}$ & 94.0662 & 94.0657 & -5.3 \\
\hline P110 & $\mathrm{C}_{6} \mathrm{H}_{8} \mathrm{NO}$ & 110.0598 & 110.0606 & 7.3 \\
\hline P191 & $\mathrm{C}_{5} \mathrm{H}_{7} \mathrm{~N}_{2} \mathrm{O}_{4} \mathrm{~S}$ & 191.0128 & 191.0127 & -0.5 \\
\hline $\mathrm{P} 284$ & $\mathrm{C}_{11} \mathrm{H}_{11} \mathrm{~N}_{3} \mathrm{O}_{2} \mathrm{SCl}^{35}$ & 284.0246 & 284.0261 & 5.3 \\
\hline $\mathrm{P} 286$ & $\mathrm{C}_{11} \mathrm{H}_{11} \mathrm{~N}_{3} \mathrm{O}_{2} \mathrm{SCl}^{37}$ & 286.0235 & 286.0231 & -1.4 \\
\hline P128 & $\mathrm{C}_{6} \mathrm{H}_{7} \mathrm{NCl}^{35}$ & 128.0259 & 128.0267 & 6.3 \\
\hline P130 & $\mathrm{C}_{6} \mathrm{H}_{7} \mathrm{NCl}^{37}$ & 130.0235 & 130.0238 & 2.3 \\
\hline P175 & $\mathrm{C}_{5} \mathrm{H}_{7} \mathrm{~N}_{2} \mathrm{O}_{3} \mathrm{~S}$ & 175.0172 & 175.0177 & 2.9 \\
\hline P495 & $\mathrm{C}_{22} \mathrm{H}_{19} \mathrm{~N}_{6} \mathrm{O}_{4} \mathrm{~S}_{2}$ & 495.0873 & 495.0909 & 7.3 \\
\hline P175 & $\mathrm{C}_{5} \mathrm{H}_{7} \mathrm{~N}_{2} \mathrm{O}_{3} \mathrm{~S}$ & 175.0172 & 175.0177 & 2.9 \\
\hline P183 & $\mathrm{C}_{12} \mathrm{H}_{11} \mathrm{~N}_{2}$ & 183.0931 & 183.0922 & -4.9 \\
\hline P497 & $\mathrm{C}_{22} \mathrm{H}_{21} \mathrm{~N}_{6} \mathrm{O}_{4} \mathrm{~S}_{2}$ & 497.0991 & 497.1066 & 15.1 \\
\hline P95 & $\mathrm{C}_{5} \mathrm{H}_{7} \mathrm{~N}_{2}$ & 95.0605 & 95.0609 & -4.2 \\
\hline P175 & $\mathrm{C}_{5} \mathrm{H}_{7} \mathrm{~N}_{2} \mathrm{O}_{3} \mathrm{~S}$ & 175.0172 & 175.0177 & 2.9 \\
\hline P185 & $\mathrm{C}_{12} \mathrm{H}_{13} \mathrm{~N}_{2}$ & 185.1068 & 185.1079 & 5.9 \\
\hline
\end{tabular}
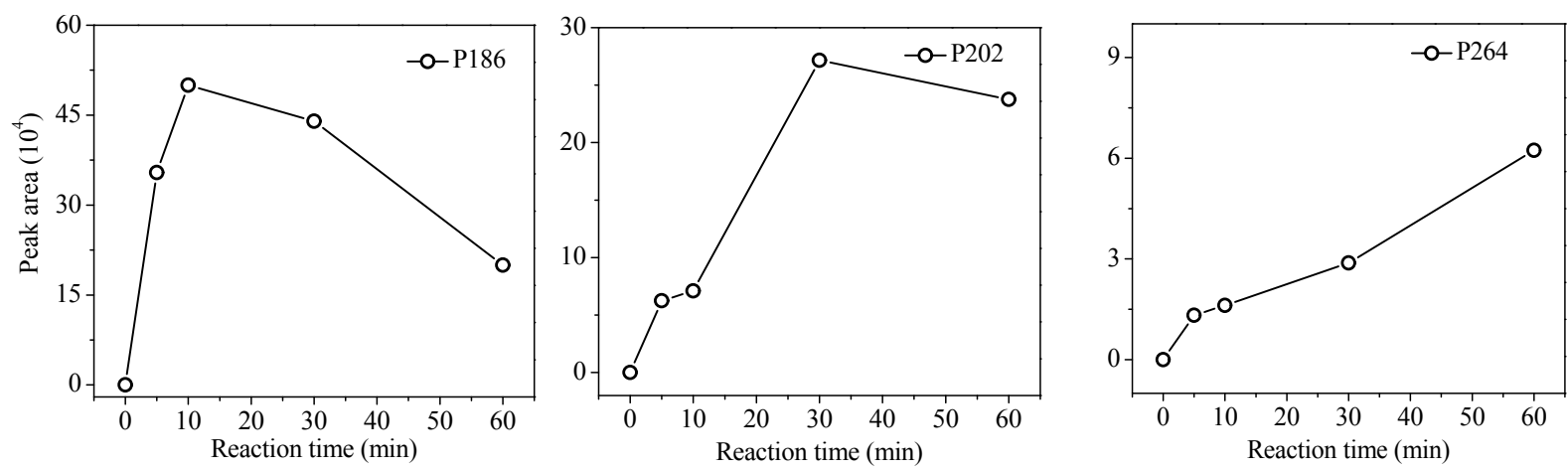

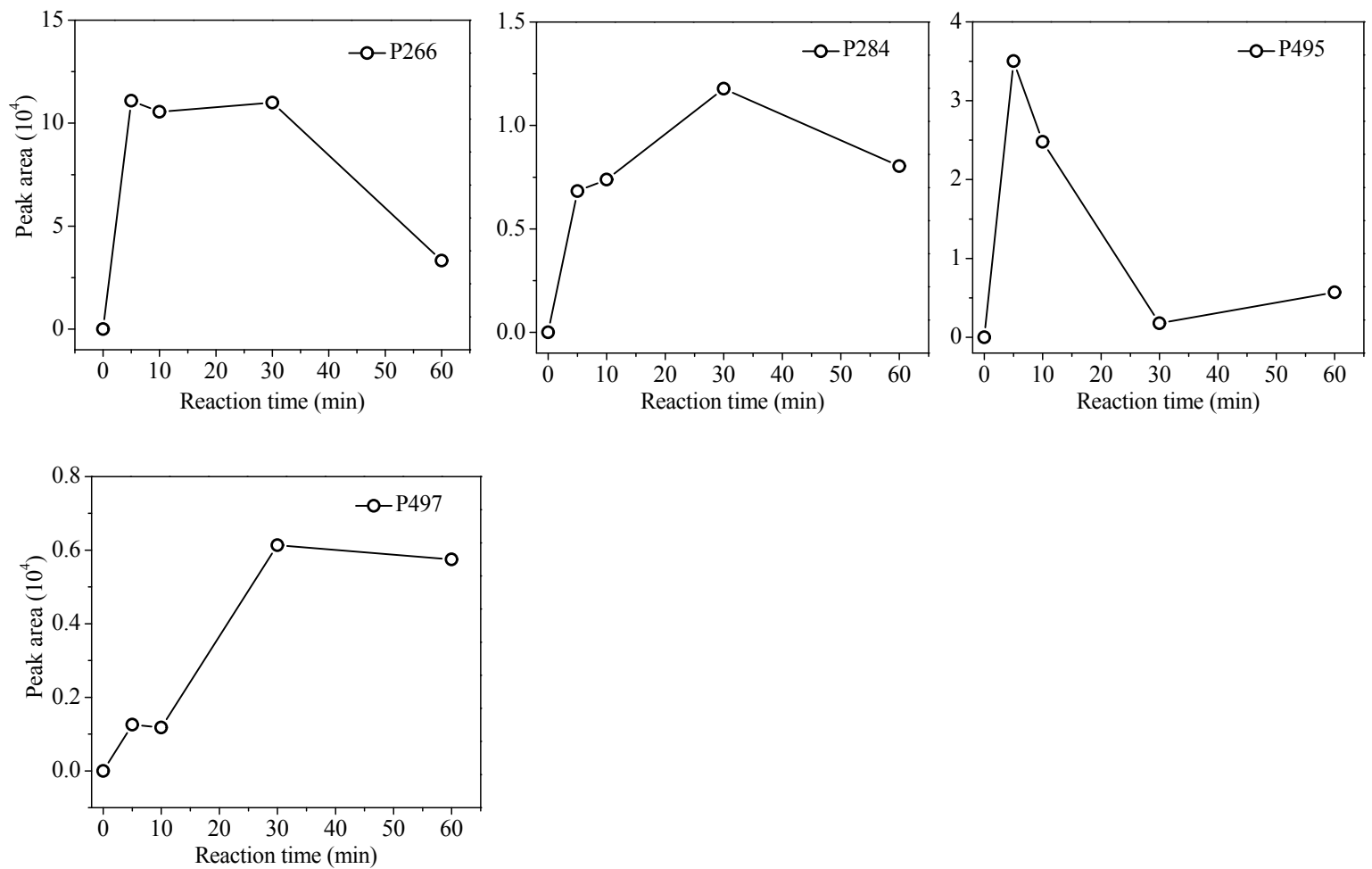

Figure S1 Evolution profiles of the degradation products of sulfapyridine under UV/chlorine system.

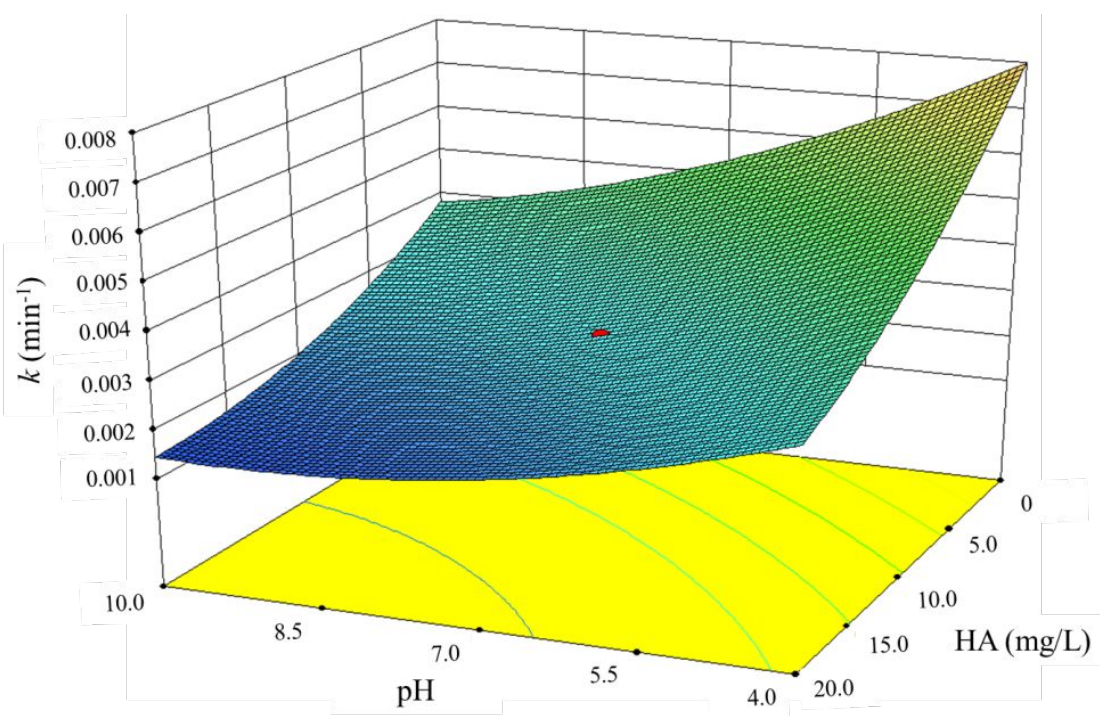

Figure S2 3D response surface graphs of pseudo-first order degradation rate constant $k$ values of sulfapyridine showing the influence of $\mathrm{pH}$ and humic acid (HA) 


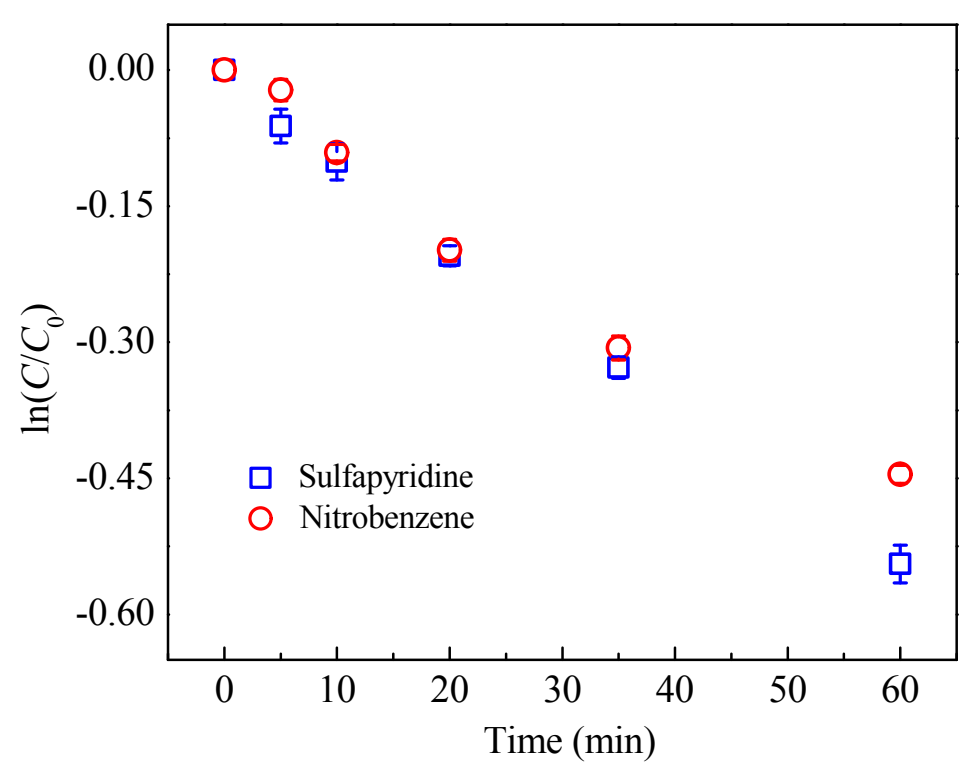

Figure S3 Degradation of sulfapyridine and nitrobenzene by $\mathrm{UV} / \mathrm{H}_{2} \mathrm{O}_{2}$ oxidation process. Experimental conditions: $\left[\mathrm{H}_{2} \mathrm{O}_{2}\right]_{0}=100 \mathrm{mM}$; $[\text { sulfapyridine }]_{0}=[\text { nitrobenzene }]_{0}=20 \mu \mathrm{M}$. Competitive kinetic reaction of sulfapyridine and nitrobenzene in $\mathrm{UV} / \mathrm{H}_{2} \mathrm{O}_{2}$ system for the determination of second-order rate constant of sulfapyridine with $\cdot \mathrm{OH}$.

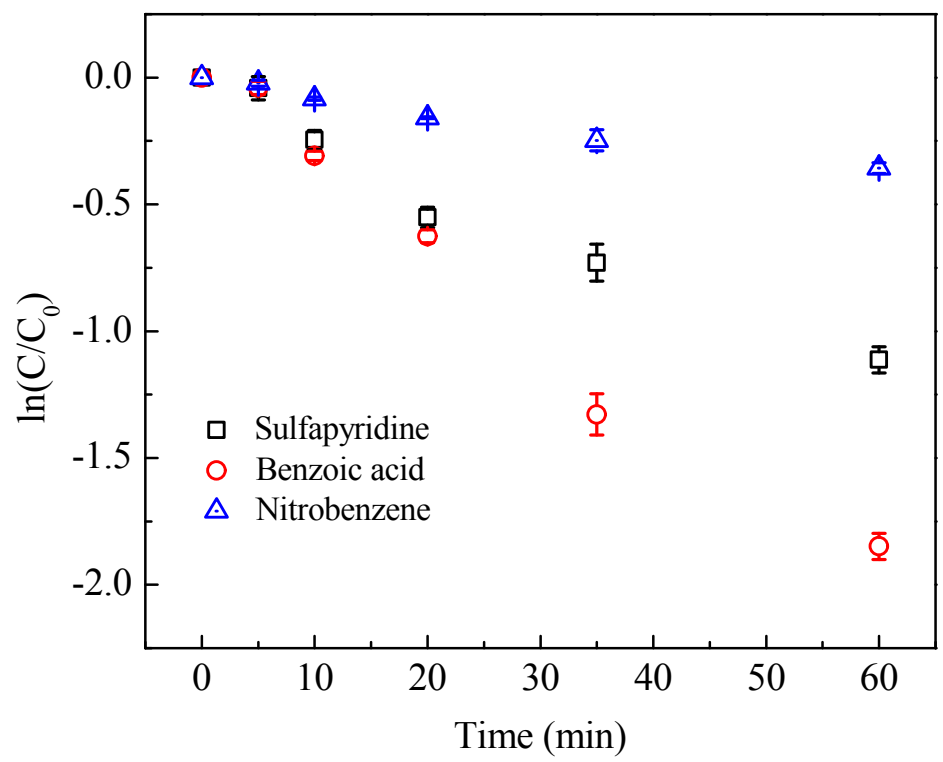

Figure S4 Degradation of sulfapyridine, benzoic acid, and nitrobenzene by UV/chlorine oxidation process. Experimental conditions: $[\text { chlorine }]_{0}=3.5 \mathrm{mg} / \mathrm{L} ;[\text { sulfapyridine }]_{0}=$ 
$[\text { benzoic acid }]_{0}=[\text { nitrobenzene }]_{0}=20 \mu \mathrm{M}$. Competitive kinetic reaction of sulfapyridine and nitrobenzene in UV/chlorine system for the determination of second-order rate constant of sulfapyridine with $\cdot \mathrm{Cl}$.

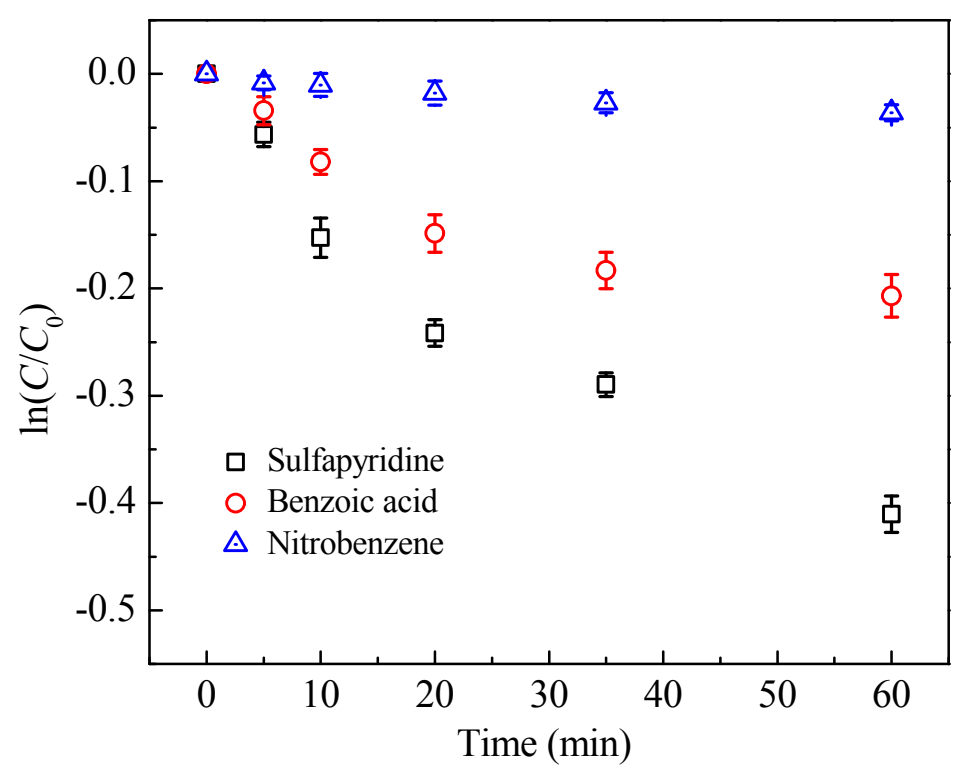

Figure S5 Degradation of sulfapyridine, benzoic acid, and nitrobenzene by UV/chlorine oxidation process containing $0.3 \mathrm{M} \mathrm{Cl}^{-}$. Experimental conditions: [chlorine $]_{0}=3.5 \mathrm{mg} / \mathrm{L}$; $[\text { sulfapyridine }]_{0}=[\text { benzoic acid }]_{0}=[\text { nitrobenzene }]_{0}=20 \mu \mathrm{M}$. 


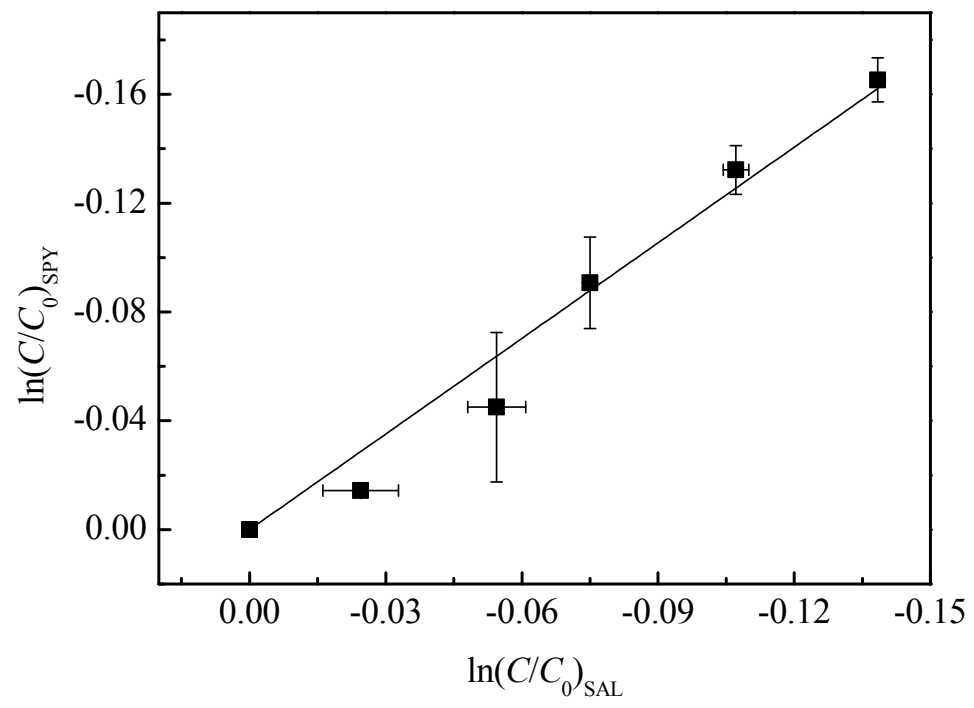

Figure S6. Kinetic plot of sulfapyridine (SPY) and salbutamol for the determination of the second-order rate constants of $\mathrm{CO}_{3}^{-} \cdot$ reacting with sulfapyridine using salbutamol (SAL) as a reference compound. Experimental conditions: $[\mathrm{SPY}]_{0}=[\mathrm{SAL}]_{0}=10 \mu \mathrm{M},\left[\mathrm{H}_{2} \mathrm{O}_{2}\right]=100 \mu \mathrm{M}$, $\left[\mathrm{HCO}_{3}^{-}\right]=100 \mathrm{mM}, \mathrm{pH}=8.4$.

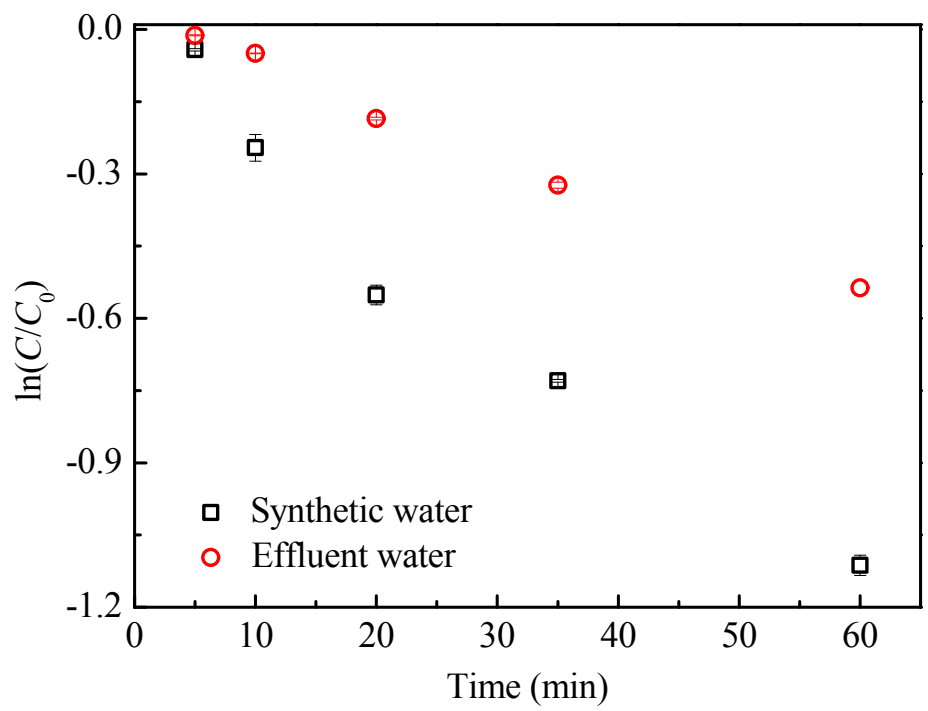

Figure S7 Degradation kinetics of sulfapyridine by UV/chlorine process in synthetic water $(\mathrm{pH}=5)$ and effluent water samples 


\section{References}

(S1) Fang, J.; Fu, Y.; Shang, C. The roles of reactive species in micropollutant degradation in the UV/free chlorine system. Environ. Sci. Technol. 2014, 48, 1859.

(S2) Kong, X.; Jiang, J.; Ma, J.; Yang, Y.; Liu, W.; Liu, Y. Degradation of atrazine by UV/chlorine: efficiency, influencing factors, and products. Water Res. 2016, 90, 15.

(S3) Wu, Z.; Guo, K.; Fang, J.; Yang, X.; Xiao, H.; Hou, S.; Kong, X.; Shang, C.; Yang, X.; Meng, F.; Chen, L. Factors affecting the roles of reactive species in the degradation of micropollutants by the UV/chlorine process. Water Res. 2017, 126, 351-360.

(S4) Cross, R.F.; Cao, J. Salt effects in capillary zone electrophoresis - III. Systematic and selective factors in the high ionic strength separation of sulphonamides in sodium phosphate buffers. J. Chromatogr. A 1998, 818, 217.

(S5) Fan, J.; Fan, Y. C.; Wang, J. J.; Cui, F. L. Extraction of aminobenzene sulfonic acid with room-temperature ionic liquids. Acta Chim. Sinica 2006, 64, 1495. 